\title{
Acordo Amazônico de Ciência, Tecnologia e Inovação em Saúde: uma experiência de integração regional
}

\author{
Edmundo Gallo, Vivian Studart, Laís Costa, \\ Sandra Willecke e Paulo Lins
}

\section{Introdução}

Em um mundo globalizado, a competitividade de uma nação está diretamente relacionada à inserção competitiva de sua região ou de seu bloco regional no cenário econômico mundial. Políticas que visem ao combate às desigualdades e à promoção do desenvolvimento socioeconômico ganham relevância crescente em âmbito regional, como requisitos capazes de impulsionar essa inserção. Vive-se um momento em que as regiões deixam de ser "suportes passivos de localização de atividades econômicas (...) assumindo um certo protagonismo na decisão de seus destinos" (DAllabrida et al., 2004). É nesse contexto que a Região Amazônica - compartilhada por nove países sul-americanos - tem seu caráter estratégico ampliado para o desenvolvimento sustentável e autonomia geopolítica, tanto para o Brasil quanto para a América do Sul.

A despeito disso, sua peculiar malha social, associada à precariedade da infra-estrutura socioeconômica, tem-se traduzido em reduzidas oportunidades 
de desenvolvimento sustentável e determinado a baixa qualidade de vida da população residente, expressa por indicadores - como o Índice de Desenvolvimento Humano $(\mathrm{IDH})-$, em sua grande maioria, abaixo da média nacional (Ministério DA SAÚde - MS, 2006: 21). A lacuna de ensino de grau superior na Amazônia, aliada à falta de oportunidade de emprego, e a conseqüente dificuldade de fixar o profissional qualificado na região têm dificultado - e até mesmo impedido - o processo de desenvolvimento regional (VAl Al, 2006). Da mesma forma, o observado "afastamento crescente entre a temática da pesquisa e as necessidades da população" (GuimarãEs, 2004) também não contribui para o enfrentamento das doenças emergentes características dos países menos desenvolvidos, impactando a capacidade de melhoria das condições socioeconômicas da população.

Segundo Becker (2004), somente "uma revolução científico-tecnológica para a Amazônia poderá promover a utilização do seu patrimônio natural em benefício da sociedade regional e nacional (...). Trata-se de implementar um novo modo de produzir com base em Ciência, Tecnologia e Inovação (CT\&I)". Esse novo olhar de C\&T para o setor social permite, inclusive, o planejamento de uma política de desenvolvimento nacional que contemple as necessidades da população brasileira.

A priorização definida pelo Governo Federal do Brasil para o desenvolvimento da Região Amazônica desencadeou uma série de articulações políticas e mobilizações que, no setor saúde, levou à realização da "1ª Oficina de Planejamento Regional Construindo uma Agenda de Saúde para a Amazônia Legal" (MS, 2003). Na ocasião, foi delineado um quadro de problemas, prioridades e estratégias para o desenvolvimento da Amazônia, além de formado um Grupo de Trabalho (GT) ampliado, com a incumbência de estabelecer as linhas gerais para a elaboração do Plano de Ações Integradas para a Amazônia Legal. Determinou-se, ainda, a realização de uma oficina sobre Ciência, Tecnologia e Inovação em Saúde (CT\&IS) na região.

A partir dessa oficina, na qual foi formado um comitê regional que apontou dificuldades, potencialidades da região e definiu uma agenda de trabalho participativa contendo políticas e programas de CT\&IS, configurou-se a necessidade de criação de um acordo interinstitucional, que resultou no estabelecimento, em 2004, do Acordo Multilateral de Cooperação TécnicoCientífica em Saúde das Instituições da Amazônia, conhecido como Acordo Amazônico de CT\&IS. Formado, originalmente, por oito instituições de ciência, tecnologia e saúde, o Acordo é composto, atualmente, por vinte e uma instituições.

O objetivo deste artigo é descrever o processo de sua organização e instalação, as ações empreendidas e seus resultados, assim como os entraves a serem superados. Enfatiza-se a importância dessa experiência em função de seu potencial de replicabilidade em âmbito regional.

Com base no levantamento de documentos oficiais do Ministério da Saúde (2003, 2006 e 2007), de reuniões do Acordo Amazônico de CT\&IS e de pesquisa sobre estudos relacionados ao tema, busca-se contextualizar o macroambiente e a importância da cooperação entre instituições brasileiras de Ciência e Tecnologia em Saúde como meio estratégico para alcançar o desenvolvimento regional da Amazônia. Apontam-se brevemente, então, alguns desafios para a região e o formato em que foi planejado 
o desenvolvimento de CT\&IS regionalizado a partir do trabalho coordenado e cooperativo. Detalhes da estrutura e evolução do trabalho também são apresentados a fim de documentar a experiência. Ademais, discorre-se sobre sua estrutura de gestão e seu estado da arte. $\mathrm{O}$ artigo é finalizado com a identificação dos resultados alcançados, assim como de suas fragilidades, incluindo algumas considerações finais.

\section{Macrodiretrizes políticas, desenvolvimento regional e Amazônia}

A Amazônia é base territorial de nove estados nacionais, com uma área de sete milhões de $\mathrm{km}^{2}$, abrigando aproximadamente $30 \%$ das florestas tropicais, a maior biodiversidade do planeta, além de um quinto do reservatório de água doce e um terço do estoque genético global - o maior banco genético terrestre, segundo dados do Plano Saúde Amazônia (Ms, 2003 e 2006). Mesmo sendo foco de disputa no cenário global - inclusive com propostas de internacionalização - a região continua carente de ações correspondentes à sua dimensão e relevância, o que é imprescindível para impulsionar seu desenvolvimento, minimizar suas desigualdades e cumprir seu papel estratégico para o alcance dos objetivos macropolíticos nacionais.

A globalização e a integração dos mercados mundiais têm suscitado preocupação entre os governantes dos países menos desenvolvidos, em função da maior vulnerabilidade a que estão expostas suas economias - comparativamente menos competitivas e menos aptas às adaptações necessárias de seu parque produtivo - e do aumento das desigualdades sociais, agravadas pela priorização da competitividade em detrimento das políticas sociais (CAssiolato Je et al., 1999). A "concorrência global passou a inserir novos padrões de qualidade, produtividade e inovação" (WiTtMann et al., 2004).

O conseqüente aumento das desigualdades, a marginalização da população e a concentração da pobreza configuram um quadro de injustiça social não condizente com a diretriz política dos estados sul-americanos, atestada por seus chefes de

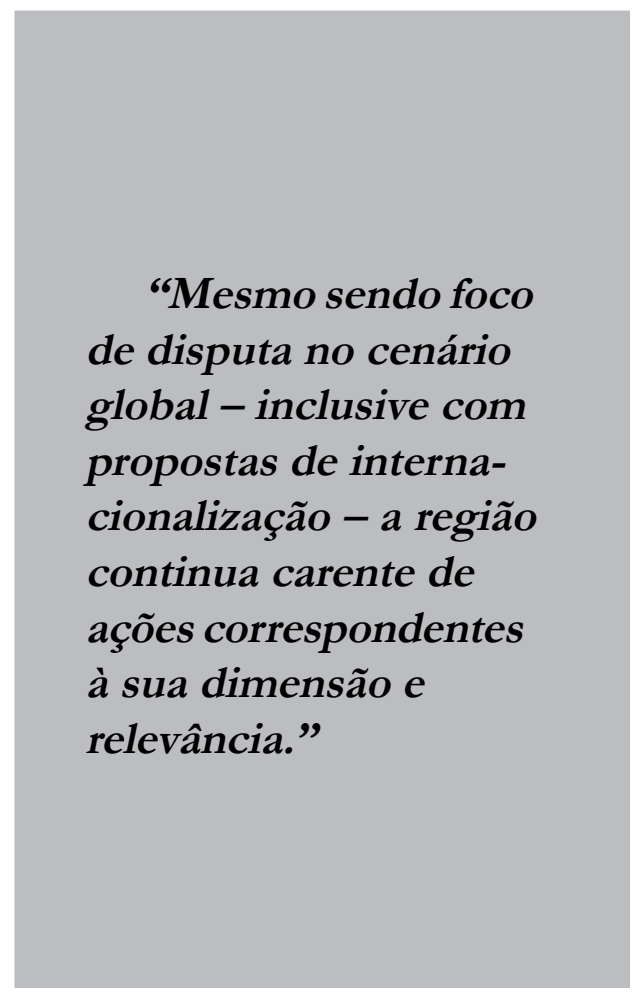

Estado, que entendem a integração do continente como um caminho para superar tal situação (Ministério DAS Relações Exteriores, 2005). Como resposta a essas 'ameaças' decorrentes da globalização, o fortalecimento de blocos e relações regionais configura-se como 'oportunidade' central para o desenvolvimento das economias mais atrasadas e para a ampliação, em bloco, da competitividade 
necessária ao desenvolvimento sustentável dessas nações (GALLO et al., 2004, 2005 e 2007).

O Plano Plurianual 2004-2007 ${ }^{1}$ reconhece isso ao eleger o desenvolvimento regional como um de seus cinco megaobjetivos. Nele, três vertentes primordiais são definidas: promoção de desenvolvimento sustentável, de cidadania e da integração sul-americana.

Sintomaticamente, a Política Nacional de Desenvolvimento Regional (PNDR) considerando, por um lado, a dimensão nacional da política e dos programas e, por outro, as desigualdades observadas entre as regiões, fossem brasileiras ou sul-americanas optou pelo foco programático sub-regional: Semi-Árido, Amazônia e Faixa de Fronteira, afirmando que a integração das zonas deprimidas é um dos desafios centrais para a desconcentração de renda, de modo que gargalos ao desenvolvimento nessas zonas têm de ser combatidos prioritariamente a partir do investimento em infra-estrutura social e econômica, necessária para a dinamização e sustentabilidade do crescimento; e o planejamento estratégico brasileiro, contemplando o formato econômico e político global vigente, deixa de ser exclusivamente nacional e passa a perseguir o fortalecimento de integração das logísticas de infra-estrutura na América do Sul, na construção progressiva de um destino comum para o continente.

Como resultado prático, sob a coordenação dos ministérios da Integração Nacional (MI) e do Meio Ambiente (MMA), foi proposto um modelo de desenvolvimento regional que estabeleceu as diretrizes gerais do Plano de Desenvolvimento Sustentável da Amazônia (PAS) e definiu cinco eixos de prioridades para a região: produção sustentável com tecnologia avançada, novo padrão de financia- mento de pesquisas, gestão ambiental e ordenamento territorial, infra-estrutura para o desenvolvimento, e inclusão social e cidadania (MI, 2006).

\section{Cooperação e integração regional}

O Ministério da Saúde (MS), no desenvolvimento do eixo "inclusão social e cidadania”, deu início à construção de uma Agenda de Saúde para a Amazônia (MS, 2003), propondo um modelo de desenvolvimento para a região sedimentado em bases social e ambientalmente aceitáveis, no qual a solução de problemas sanitários mais críticos foi considerada como um dos pressupostos básicos.

Foi realizada na cidade de Manaus, em agosto de 2003, a $1^{\text {a }}$ Oficina de Planejamento Regional - Construindo uma Agenda de Saúde para a Amazônia Legal. A iniciativa embasou-se metodologicamente no fato de que "as diferentes dinâmicas de desenvolvimento regional dependem de uma crescente organização social das comunidades regionais, o que equivale, diretamente, a um melhor envolvimento político nas decisões e definições dos rumos do desenvolvimento regional, assim como a um maior desenvolvimento econômico das comunidades regionais" (BECKER, 2003).

O resultado final da oficina foi a constituição de um quadro de problemas, prioridades, estratégias/ações, atores responsáveis e a formação de um Grupo de Trabalho (GT) ampliado ${ }^{2}$, com a tarefa de integrar os atores e as instituições da Região Amazônica, configurando um mecanismo de articulação direta entre o MS e os poderes regionais.

O GT elaborou o Plano de Qualificação da Atenção à Saúde na Amazônia Legal: Plano Saúde Amazônia (2), que 
determinava ao Ministério da Saúde a "formulação de propostas que viabilizassem a adequação do SUS na Amazônia Legal, aproveitando o potencial da biodiversidade local para desenvolver sustentavelmente as comunidades". Para tanto, o GT teve por atribuição "conduzir o processo de articulação interna e externa e desenvolver as estratégias necessárias, contando com a participação de representantes das diferentes áreas do MS" (MS, 2003), considerando a "necessidade de mudança na condução das inter-relações com a região, sobretudo no processo de identificação de problemas e construção de estratégias de intervenção. A partir disso, a idéia da construção de um Plano com a região superou a dimensão da formulação de um Plano para a região" (Ms, 2003).

Em outubro de 2003, outra oficina de trabalho foi realizada em Belém com o tema "Ciência, Tecnologia e Inovação em Saúde na Região Amazônica", objetivando um maior detalhamento e pactuação das prioridades de pesquisa e ensino em saúde na região e a definição de um conjunto de ações a serem valorizadas. De acordo com o relatório resultante da oficina, o grupo discutiu, entre outras questões, problemas relacionados à formação de recursos humanos em Ciência, Tecnologia e Inovação em Saúde (CT\&IS), à informática e informação em saúde, à situação da inovação tecnológica em saúde e à necessidade de investimentos e definição de prioridades de pesquisa em saúde (Ms, 2003).

Foi formado um comitê regional composto por instituições de ensino, pesquisa e serviços de saúde, cujas proposições incluiriam a identificação de potencialidades e dificuldades da região, assim como a construção de uma agenda de trabalho que estabelecesse a definição participativa de políticas e programas da área.

Ainda segundo o relatório, além dos grupos temáticos específicos foi organizado outro que buscou discutir e elaborar as macroestratégias necessárias para o desenvolvimento das prioridades que seriam definidas no decorrer da oficina ${ }^{3}$. No âmbito desse grupo foi aprovada a proposta - apresentada pela Fiocruz - de formação de um Acordo Multilateral de Cooperação entre as Instituições Técnico-científicas da Amazônia - Acordo Amazônico de CT\&IS, que visava ao "desenvolvimento de programas, projetos e atividades no campo da investigação conjunta para conhecer as realidades sociossanitárias e epidemiológicas da Amazônia e as respostas do Sistema de Saúde e de Vigilância Epidemiológica” (MS, 2004).

\section{Acordo Amazônico de Ciência, Tecnologia e Inovação em Saúde}

A demanda de articulação entre as ações de fomento científico, tecnológico e de saúde é necessária para melhorar a "capacidade de transferência de conhecimento novo para as indústrias, serviços de saúde e para a sociedade em geral" (Guimarães, 2004), conformando um modelo de desenvolvimento sustentável e inclusivo. Além da aprovação e constituição do Acordo Amazônico, foram discutidas e elaboradas as estratégias organizativas e ações que deveriam ser desenvolvidas, visando à sua efetiva implementação. Adicionalmente, foi aprovada a formação de um Conselho Diretor, composto pelos dirigentes máximos das instituições partícipes ou seus representantes indicados, e cuja atribuição seria a definição de estratégias e resoluções conjuntas (Ms, 2003). 
O plano de ação para o Acordo Amazônico contemplou a estruturação de uma secretaria executiva que promovesse comunicações e debates, facilitando a interação dos participantes, que conduzisse a elaboração dos documentos dos GTs para apresentação nas reuniões subseqüentes, que mapeasse instituições passíveis de convocação para a referida reunião, e que promovesse a constituição de um Conselho Gestor a partir das indicações dos dirigentes das instituições.

Em princípio, foram identificadas como prioridades para o Acordo: a constituição de redes cooperativas de projetos, linhas e programas de pesquisa e desenvolvimento (P\&D), o incentivo à cooperação técnica com o SUS, a valorização da participação das instituições científicas da região na melhoria das atividades de vigilância em saúde e o apoio à melhoria da qualificação de docentes e pesquisadores. Quanto à definição de temas estratégicos, foram eleitos GTs de Pesquisa e Desenvolvimento, Recursos Humanos e Infra-estrutura. Projetos de trabalho em cada uma das áreas deveriam ser elaborados pelos GTs com $\mathrm{o}$ intuito de dar concretude às diretrizes estabelecidas e com o objetivo de integrar as ações realizadas pelas diversas instituições e pesquisadores filiados ao Acordo Amazônico.

O relatório da $2^{\text {a }}$ Oficina do Acordo (Ms, 2005), realizada na cidade de Porto Velho (RO), em dezembro de 2003, mostra que as discussões do GT de Pesquisa e Desenvolvimento em Saúde na Amazônia possibilitaram identificar ações consideradas essenciais para garantir o processo de consolidação do Acordo e as demais atividades relacionadas à busca e à oferta de financiamento de projetos de pesquisa por meio de edital lançado pelo
Departamento de Ciência e Tecnologia (Decit) do Ministério da Saúde, em 2004. Esse edital relacionou como prioritários os seguintes temas e subtemas: doenças associadas a vetores (malária, leishmaniose, arboviroses); doenças não associadas a vetores (tuberculose, hanseníase, hepatites virais, diarréias); bioativos de produtos naturais; pesquisa em sistemas de serviços de saúde; desenvolvimento, ambiente e saúde; e outros agravos e doenças relevantes.

Definiu-se, ainda, que os temas e subtemas propostos seriam discutidos com o corpo de pesquisadores das instituições parceiras de modo a obter consenso sobre as prioridades estabelecidas na reunião. Posteriormente à sua definição, os temas deveriam ser trabalhados em redes de pesquisas que congregassem diversas instituições regionais. Ademais, as instituições deveriam contribuir para a elaboração dos editais que viriam a ser publicados pelo Decit/MS (MS, 2005).

O GT de Recursos Humanos discutiu a formação de cursos de pós-graduação stricto sensu, propondo que os programas de pós-graduação para a Amazônia devessem ser entendidos como uma ação estratégica e diferenciada de formação de recursos humanos em Ciência e Tecnologia em Saúde, requerendo, portanto, tratamento diferenciado pelos órgãos de avaliação.

Indo ao encontro da $2^{\text {a }}$ Conferência Nacional de Ciência, Tecnologia e Inovação em Saúde ( $2^{\mathrm{a}}$ CNCTIS), que reconhece a necessária "interação estreita entre o Sistema Único de Saúde, os componentes de C\&T e a política de formação de recursos humanos em saúde" (Ms, 2004), o grupo sugeriu que o Acordo Amazônico promovesse a identificação da capacidade instalada na região; a busca de mecanismos de reforço à capacidade institucional de 
pós-graduação na Amazônia; a construção e a implementação de novos cursos de mestrado e doutorado em Ciências da Saúde com prioridade em Saúde Coletiva, Doenças Infecciosas e Parasitárias especialmente Biologia Celular e Molecular - e em Saúde e Ambiente; e, por fim, mestrado profissional nas áreas de Clínica e Epidemiologia.

A proposta de um modelo para pós-graduação na Amazônia deveria basear-se em uma rede multiinstitucional com núcleos de execução a serem definidos após o levantamento da capacidade instalada e a grade curricular, a qual deveria conter o número mínimo de disciplinas comuns, presenciais, realizadas nos núcleos de execução, e uma grade complementar, descentralizada, com a orientação de dissertações e teses em regime tutorial. $\mathrm{O}$ aprofundamento do diagnóstico da capacidade instalada em pesquisa e ensino na Amazônia foi definido como tarefa da Secretaria Executiva do Acordo, que apresentou o resultado na Oficina do Acordo Amazônico, em Rio Branco (AC), em maio de 2004.

O principal objetivo dessa oficina foi garantir a formulação dos subsídios necessários à elaboração do edital para financiamento de projetos de pesquisa, lançado pelo Decit/MS, bem como aprofundar a discussão sobre a organização de programas de pós-graduação capazes de contemplar as necessidades da região.

Os participantes da oficina foram divididos em grupos de trabalho para o aprofundamento dos debates sobre as estratégias a serem seguidas na constituição das oito redes temáticas ${ }^{4}$. Reconheceu-se ainda a necessidade de revisão e alteração das exigências da Capes em relação aos critérios de avaliação das propostas de novos cursos de pós-graduação que atendessem às demandas e especificidades da Região Amazônica, sugerindo-se que os signatários do Acordo Amazônico reivindicassem essa revisão. Reafirmou-se a importância da articulação entre os cursos propostos e os núcleos de pesquisa já existentes, e propôs-se uma nova reunião do grupo de RH com todos os representantes institucionais para aperfeiçoar a

“ $O$ conseqüente

aumento das

desigualdades, a

marginalização

da população e a

concentração da

pobreza configuram

um quadro de injustiça

social não condizente

com a diretriz política

dos estados sul-

americanos."

proposta apresentada pelo grupo na reunião do Conselho Gestor.

Acordo Amazônico de CT\&IS: estado da arte

Formalizado em 26 de outubro de 2004, com sua publicação no Diário Oficial da União (seção 3, no 206, anexo 5), o 
Acordo Amazônico é composto atualmente de 21 instituições ${ }^{5}$.

Com o objetivo de organizar e garantir a execução das ações traçadas, o Acordo Amazônico adotou, desde 2006, a seguinte estrutura organizacional: o Conselho Diretor, composto por todos os dirigentes das instituições que integram o projeto, tem a missão de avaliar anualmente o planejamento estratégico e de deliberar sobre o período subseqüente; o Conselho Gestor - constituído por dois representantes do eixo ocidental (AM, AC, RR e RO), dois do eixo oriental (PA, AP e TO) e dois do MS (Decit e DAD) -, tem o papel de implantar, monitorar e avaliar o Plano de Ação do Acordo, com o mandato limitado até a próxima reunião do Conselho Diretor; a Secretaria Executiva, formada pelo Centro de Pesquisa Leônidas e Maria Deane (CPqLMD/Fiocruz), tem a função de fornecer suporte operacional às atividades do Acordo; e a Coordenação Geral - representada pela Fiocruz/Brasília é responsável por coordenar as atividades do Acordo.

No início de 2006, foi realizada a reunião do Conselho Diretor, que traçou o plano de trabalho para o ano que se iniciava, avaliando as ações realizadas até aquele momento e as prioridades necessárias. Ao longo do ano, ocorreram cinco reuniões do Conselho Gestor para monitoramento, avaliação e redefinição de ações e resultados esperados.

Desde o seu estabelecimento até o presente momento, o Acordo Multilateral assegurou o aumento de eficiência em várias ações de CT\&IS na Região Amazônica. Diversos empecilhos também puderam ser observados. A seguir, listarse-ão os resultados alcançados, seguidos das principais conquistas e dificuldades enfrentadas.

\section{Resultados alcançados}

\section{Formação das redes temáticas}

De acordo com Castells (1999), as "redes de cooperação tecnológica facilitam a aquisição de tecnologia para projetos e produção de produtos, capacitam o desenvolvimento conjunto dos processos e da produção e permitem acesso compartilhado a conhecimentos científicos genéricos e de P\&D”. Nessa perspectiva, foram propostas oito redes temáticas, sendo sete relacionadas à pesquisa, desenvolvimento e inovação tecnológica em saúde ${ }^{6}$ e uma de ensino, com ênfase na formação de recursos humanos em nível de mestrado e doutorado.

Conforme a orientação do Ministério da Saúde e de acordo com a previsão inicial da Oficina do Acre, cada uma dessas redes teria o objetivo de desenvolver atividades de pesquisa científica, tecnológica e inovação na sua área de atuação específica.

\section{Editais de pesquisa com base nas prioridades do Acordo Multilateral}

A partir da constituição dessas redes, as oficinas do Acordo Amazônico propiciaram um amplo diálogo entre os pesquisadores e representantes das instituições partícipes, possibilitando o estabelecimento das áreas prioritárias contempladas no edital lançado pelo Decit/MS em 2004.

O Programa "Pesquisa para o SUS PPSUS/Saúde Amazônia” do Decit/MS, que compreende o fomento a pesquisas em saúde e o fortalecimento do SUS na Amazônia Legal, definiu como áreas prioritárias aquelas correspondentes às sete redes temáticas de pesquisa do Acordo Amazônico citadas. Importa enfatizar que 
$70,8 \%$ dos projetos aprovados são provenientes de instituições e/ou pesquisadores vinculados ao Acordo, com destaque para as redes temáticas Síndromes Fébris Ictero-hemorrágicas Agudas, Fitoterápicos e Micobacterioses.

\section{Curso de mestrado em Saúde, Sociedade e Endemias na Amazônia}

Iniciada durante a reunião realizada na cidade de Porto Velho, e a partir das considerações acerca do processo de análise das necessidades de cursos de pósgraduação na área de saúde a serem implantados na Região Amazônica, a proposta de organização do curso de mestrado em Saúde, Sociedade e Endemias - organizado em rede por meio da parceria entre a Universidade Federal do Pará (UFPA), a Universidade Federal do Amazonas (UFAM) e o CPqLMD/ Fiocruz - foi aprovada pela Capes em março de 2005.

A primeira turma, composta por 28 alunos, começou já no segundo semestre de 2005. Para setembro de 2007/ $1^{\circ}$ semestre de 2008 estava prevista a defesa das dissertações para conclusão do curso. No segundo semestre de 2006, teve início a segunda turma do curso de mestrado, prevista para terminar em 2008.

\section{Curso de doutorado em Saúde Coletiva}

Considerando que a carência de doutores na área de Saúde Coletiva na Região Amazônica tem limitado as atividades de pesquisa no campo da saúde e impedido a abertura de pós-graduação stricto sensu nessa área, foi implantado na Fiocruz do Amazonas, em junho de 2005, o curso de doutorado em Saúde Coletiva.
Pautado por uma visão integradora e totalizante do processo formador, o curso de doutorado pretende contribuir com a estruturação de cursos permanentes de pós-graduação stricto-sensu na região. Dessa forma, a definição da grade curricular e de projetos de pesquisa é norteada pelas prioridades do mestrado de Saúde, Sociedade e Endemias na Amazônia, uma vez que se pretende que os 15 doutores formados no curso venham a ingressar em seu quadro docente, reforçando-o.

\section{Elaboração de proposta para acreditação dos hospitais de ensino}

Um dos desafios traçados para o ano de 2006 pelo Conselho Diretor do Acordo foi a adequação dos cursos de graduação da Região Amazônica à realidade do Sistema Único de Saúde, visando à promoção da formação de recursos humanos mais bem preparados para atender à demanda local e regional dos serviços de saúde. Com esse objetivo, foi articulado um grupo de trabalho para identificação das políticas vigentes nos hospitais, resultando na elaboração de uma proposta de acreditação diferenciada para os hospitais de ensino da região, que previa incentivos vinculados ao alcance de metas de qualidade.

\section{Rede Pan-Amazônica de Ciência, Tecnologia e Inovação em Saúde}

O trabalho em rede é considerado por Levy (2004) e Cavalcanti e Nepomuceno (2007) como um novo modo de produção do conhecimento, no qual a tradicional e necessária identificação dos desafios e problemas, bem como o esforço para superá-los, oportuniza-se a partir da interação, em tempo real, de uma 
série de especialistas complementares entre si, além de usuários; enfim, uma série de atores agregados em redes de cooperação com finalidades comuns. Por sua vez, de acordo com o Centro de Gestão e Estudos Estratégicos - CGEE (2006:8), a gestão em rede "enfatiza a cooperação, a interação, a redução das entropias e o funcionamento ou o desenvolvimento das atividades como um sistema aberto e altamente dinâmico". Segundo Cavalcanti e Nepomuceno, a produção de inteligência coletiva pressupõe comunidades articuladas em rede, regularidade e conhecimento dos membros, interação consciente desses nós, além de uma estrutura e ferramenta de gerenciamento e trabalho para os profissionais do conhecimento, da informação e da comunicação (CAVALCANTI et al., 2007: 40).

O Acordo Amazônico propôs a implantação de uma Rede Cooperativa das Instituições de Ciência, Tecnologia e Inovação em Saúde abrangendo os diversos países que compõem a Região Amazônica (Bolívia, Brasil, Colômbia, Equador, Guiana, Peru, Suriname e Venezuela ${ }^{7}$ ). O projeto reconhece a importância do desenvolvimento de uma cultura empreendedora que priorize o enfoque regional, assim como a importância de se promover a inteligência cooperativa, sendo que esse processo não pode restringir-se a um país isoladamente. Adicionalmente, essa rede responde à "reivindicação por parte das populações amazônicas sul-americanas de uma melhor interconexão entre os diversos países pelas redes de circulação, de modo a criar e intensificar redes de relações de todo tipo" (Machado, 2003).

Denominado como marco inicial do trabalho da Rede Pan-Amazônica, o Encontro Internacional Pró-Rede
Pan-amazônica de CT\&IS foi realizado na cidade de Manaus (Amazonas), em novembro de 2006. Seus resultados podem ser observados na publicação bilíngüe "Rede Pan-amazônica de Ciência, Tecnologia e Inovação em Saúde: uma proposta estratégica de integração" (Ms, 2007).

As diretrizes políticas da formação da rede encontram-se resumidas na Carta de Manaus . Essa carta reafirma, entre outros itens, "a importância geopolítica estratégica da Amazônia e seu potencial de contribuição para o desenvolvimento sustentável dos países amazônicos, assim como da humanidade e sobrevivência do planeta; a importância do desenvolvimento científico e tecnológico voltado para a inovação como instrumento de promoção da eqüidade, qualidade de vida e saúde da população; (...) a integração dos países da região como caminho para o desenvolvimento humano e sustentável e a justiça social; a importância de políticas transnacionais e intersetoriais articuladas para o alcance das metas do milênio; a necessidade de ações que promovam sistemas de saúde universais e equânimes, respeitando as diferentes culturas dos povos da região; e a urgência de se construir e implantar políticas e mecanismos eficazes que melhorem a excelência técnica das instituições de pesquisa e das formadoras de recursos humanos que contribuam na diminuição de desigualdades, e que promovam compromisso solidário com as gerações futuras" (MS, 2007).

O II Encontro Internacional foi realizado entre os dias 9 e 11 de julho de 2007, em Belém (PA), e representou um avanço na implantação da rede, proporcionando definições estratégicas para o trabalho e um diálogo mais articulado entre seus componentes. 
Os resultados desse II Encontro foram a definição do modelo de gestão e das instituições/atores responsáveis; a identificação dos principais temas relacionados à CT\&IS que deverão compor a agenda comum; a identificação dos recursos críticos (políticos, sociais, humanos, físicos e financeiros) relacionados à sustentabilidade da Rede Pan-Amazônica; e o apoio político e o compromisso de cada país e instituições multilaterais na implantação e sustentabilidade da rede.

O documento político de intenções resultante - a Carta de Belém - ressalta:

"Os países: Bolívia, Brasil, Colômbia, Equador, Peru, Suriname e Venezuela, reunidos na cidade de Belém (Brasil), aos onze dias de julho de 2007, como integrantes da Rede Pan-amazônica de CT\&IS, (...)

\section{CONCORDAM EM: (...)}

- Incentivar e apoiar políticas públicas voltadas para o desenvolvimento de CT\&IS e orientadas para a busca de alternativas de melhoria da situação da saúde em cada uma das suas regiões, dentro de uma perspectiva ecossistêmica, social, política, cultural e econômica;

- Privilegiar a incorporação da população amazônica nos processos produtivos e nos benefícios resultantes das inovações geradas em decorrência do desenvolvimento de CT\&IS na região;

- Priorizar o desenvolvimento dos recursos humanos em Ciência, Tecnologia e Inovação em Saúde na Região Amazônica, observando os Acordos de Toronto de 2005" (sítio da Rede Pan-Amazônica, 2003).
A rede tem entre seus objetivos promover o desenvolvimento e o fortalecimento da Ciência, Tecnologia e Inovação em Saúde, não somente em nível nacional, mas principalmente em nível regional, incluindo, para tanto, os países que compõem a Região Amazônica. Busca o desenvolvimento sustentável da região, com formato inclusivo, valorizando o potencial humano e socioeconômico e melhorando a qualidade de vida da

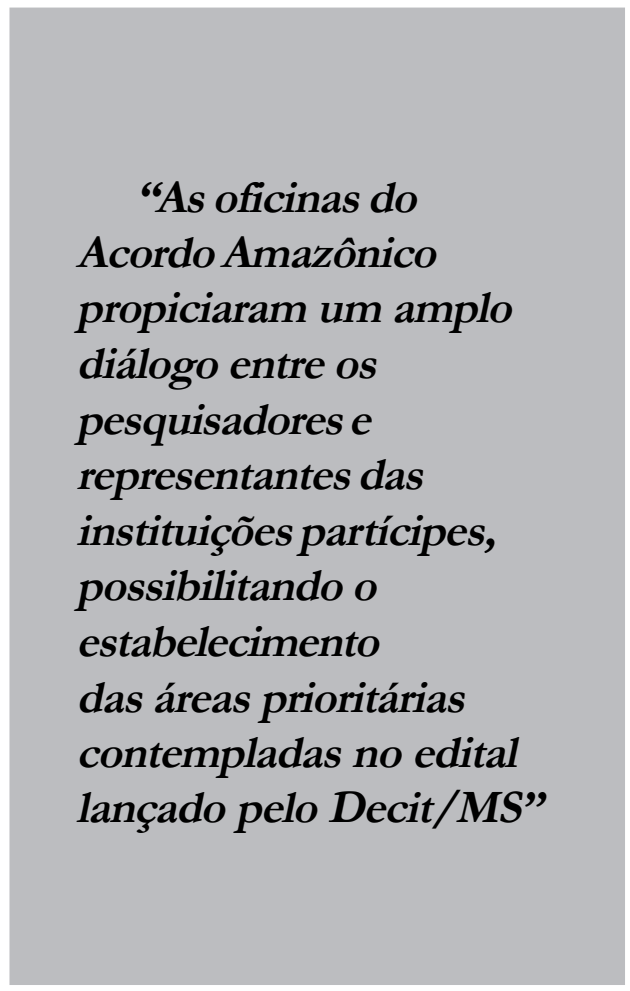

população. Pretende alcançar esse resultado por meio da proposição e apoio à implementação de macropolíticas convergentes que possibilitem a integração de ações que objetivem a expansão de cadeias produtivas, pólos tecnológicos, investigações e experiências de gestão e cooperação técnica voltadas à produção de insumos estratégicos, à promoção e gestão de 
sistemas de saúde universais e equânimes, à educação permanente e ao desenvolvimento sustentável da Amazônia.

O processo de implantação dessa rede envolve um trabalho executivo, que vem sendo realizado de modo cotidiano pela Organização do Tratado de Cooperação Amazônica (OTCA), pela Organização Pan-Americana de Saúde (OPAS) e pela Fundação Oswaldo Cruz (Fiocruz). Envolve um processo deliberativo de integração, já que as decisões relativas à rede são pactuadas entre os países, o meio acadêmico e os governos nacionais e subnacionais. As reuniões deliberativas ocorrem periodicamente e até o momento já foram realizados dois encontros internacionais, um curso sobre gestão e trabalho em redes, além de inúmeras reuniões de sua secretaria técnico-operativa.

\section{Sucessos e dificuldades}

Os esforços empreendidos até o momento são grandes e produziram resultados positivos, assim como apontaram para fragilidades que precisam ser superadas.
Vale notar a participação expressiva nos editais promovidos pelo Ministério da Saúde, por intermédio do Departamento de Ciência e Tecnologia (Decit) da Secretaria de Ciência, Tecnologia e Insumos Estratégicos (editais do programa "Pesquisa para o SUS: gestão compartilhada em Saúde - PPSUS"), dos pesquisadores ligados ao Acordo, cujas atividades contribuíram de forma significativa para incrementar o aporte de recursos para o desenvolvimento de pesquisas na Amazônia. De 2004 para 2005, o número de projetos de pesquisa apoiados pelo Decit aumentou em 47\%, mas o volume de recursos despendido foi bem mais significativo, tendo sido majorado em 309\% (MS, 2007).

$\mathrm{Na}$ realidade, o total de recursos liberado pelo Decit, complementado pelas fundações de amparo à pesquisa em alguns estados, e apropriado pelos pesquisadores das diversas unidades federadas da Região Amazônica, ultrapassou o teto de $\mathrm{R} \$ 4$ milhões (MS, 2005).

Como ponto débil a ser superado, destaca-se que, a despeito da polarização de esforços em torno dos editais do Decit

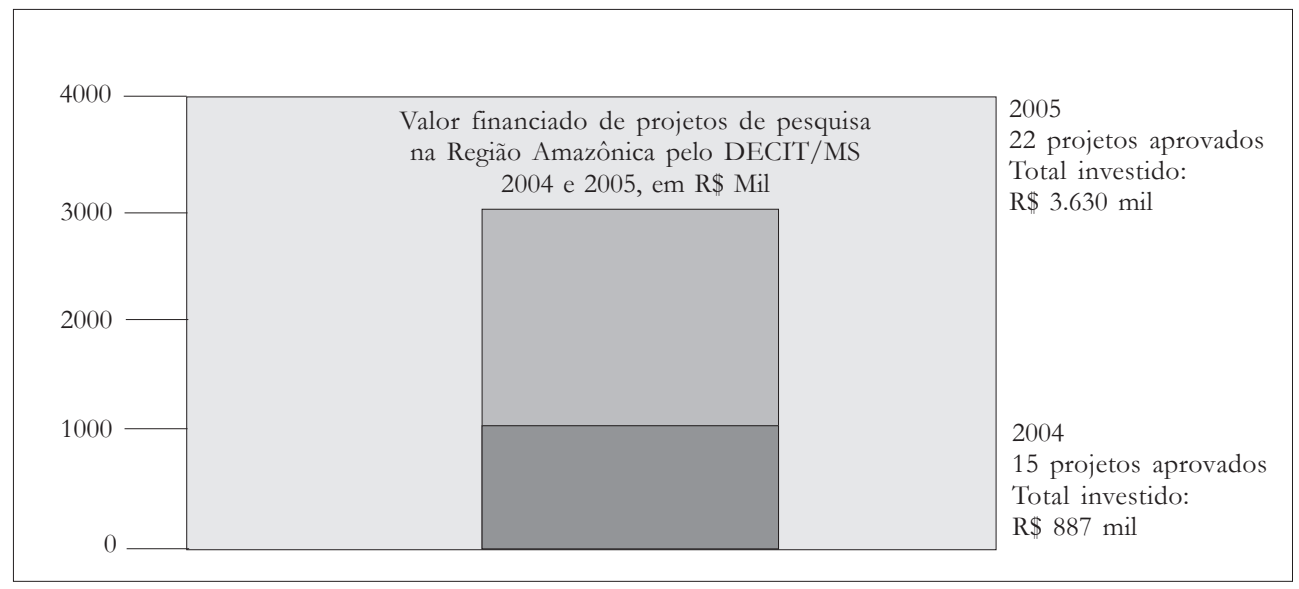

Fonte: Elaboração própria a partir de dados do Brasil (MS, 2005). 
ter favorecido a mobilização dos pesquisadores e gestores pela iniciativa, à medida que os editais eram lançados e os projetos aprovados tal mobilização decrescia, já que a principal motivação para participar das ações do Acordo estava ligada à busca de recursos para pesquisa. Ademais, os editais do Decit, apesar de favoráveis ao incentivo à pesquisa, pouco contribuíram para o estímulo à pesquisa em redes, uma vez que propunham financiamentos por unidade federada.

Como um ponto crítico positivo, deve-se notar que as atividades do Acordo tornaram viáveis diversas formas de interação de pesquisadores e gestores das instituições signatárias, contribuindo com o debate democrático e gerando discussões e propostas de redirecionamento dos rumos da pesquisa e da pós-graduação na Amazônia. A riqueza desses momentos demonstrou a potencialidade para o futuro desenvolvimento de uma política regional integrada de ensino e pesquisa de C\&T em saúde, o que acabou apontando para a formação da Rede Pan-amazônica de CT\&IS, já implantada e politicamente articulada entre os países.

É importante, entretanto, não ignorar que apesar desse relativo incremento da capacidade organizativa e do crescimento das ações conjuntamente pactuadas entre as instituições de ensino e pesquisa na Amazônia, isso ainda é insuficiente em relação à proposta do Acordo.

A paralisação das atividades, devido ao comprometimento insuficiente dos representantes com a proposta do Acordo e à dificuldade de sustentabilidade financeira, ocorrida entre a reunião do Acre e a do Conselho Diretor em fevereiro de 2006, seguida da redução das atividades entre dezembro de 2006 e outubro de 2007, fragilizou a credibilidade e legitimidade da iniciativa junto às instituições amazônicas. Tal situação terá de ser revertida a partir de um esforço programado de retomada das articulações com os gestores das instituições signatárias.

Esse comprometimento "falho" não é peculiaridade da relação das instituições signatárias do Acordo. Guarda coerência com a parca articulação política das instituições amazônicas, que carecem de um programa regional articulado e capaz de intervir positivamente nas instâncias nacionais de fomento e na promoção à produção científica para a região, em busca de fazer valer suas demandas e necessidades. Esse é, na realidade, um ponto significativamente crítico a ser superado.

No âmbito da gestão da política de CT\&IS, o desenvolvimento das atividades do Acordo possibilitou a obtenção de uma visão panorâmica sobre as condições político-institucionais da produção científica na região, assim como produziu maior identificação entre as instituições partícipes. Esse empoderamento ${ }^{8}$ regional pode ajudar, no futuro, na superação de dificuldades de integração entre os coordenadores das redes temáticas e no estabelecimento de premissas e prioridades dos gestores das signatárias - preocupados em gerar políticas públicas de aprimoramento do ensino e pesquisa (MS, 2005). Diversas redes de pesquisa são preexistentes ao Acordo e foram por ele potencializadas. A lógica, entretanto, que rege a articulação dos pesquisadores, é distinta daquela que deve reger as iniciativas articuladas dos gestores para gerar políticas de C\&T em Saúde. Essas distintas lógicas em interação não dispuseram de tempo hábil para ser harmonizadas segundo as premissas do Acordo.

Por fim, um dos sucessos do Acordo que vale menção foi a efetivação dos cursos 
de mestrado multidisciplinar em Saúde, Sociedade e Endemias e de doutorado em Saúde Coletiva. A interação propiciada pelo Acordo foi a mola propulsora da articulação institucional firmada entre o Centro de Pesquisas Leônidas \& Maria Deane, a Universidade Federal do Pará, a Universidade Federal do Amazonas, a Vice-Presidência de Ensino e Comunicação, a ENSP, a Aggeu Magalhães e o Instituto Fernandes Figueira, que possibilitou, em um processo relativamente rápido, a implantação dos dois cursos.

\section{Considerações finais}

Atualmente, a insuficiência de recursos financeiros, a desarticulação dos investimentos, a precária organização das instituições executoras e fomentadoras de pesquisa e desenvolvimento, a ineficiência dos mecanismos de planejamento, financiamento, gerenciamento e avaliação da pesquisa e a pouca familiaridade com o sistema de propriedade intelectual são algumas das barreiras ao desenvolvimento da Amazônia. A baixa capilaridade das instituições acadêmicas explica, em parte, a desarticulação das iniciativas entre si e a escassez de recursos voltados para trabalhos articulados com as políticas nacionais e regionais. A duplicação de esforços e a conseqüente dificuldade de alcançar os fins desejados marcam a atuação da Ciência, Tecnologia e Inovação em Saúde, contribuindo para o insuficiente desenvolvimento da região.
A consolidação do Acordo Amazônico de CT\&IS busca incentivar a geração de CT\&I em Saúde, pressuposto básico para superar as condições críticas de saúde da região e reduzir as desigualdades regionais do País, constituindo, portanto, uma iniciativa extremamente relevante.

Apesar das peculiaridades de cada estado amazônico, questões comuns são partilhadas por toda a região, assumindo, assim, uma extensão regional em seus efeitos socioeconômicos e sanitários. Desafios comuns podem ser mais bem estudados e desenvolvidos de forma coordenada e colaborativa. Ademais, a escassez de recursos especializados, além dos financeiros, sugere o aproveitamento da sinergia de trabalho comum coordenado para solucionar questões igualmente partilhadas. Após o conhecimento das potencialidades, o planejamento do desenvolvimento conjunto em bases sustentáveis tende a beneficiar a região como um todo. O enfoque regional fortalece as capacidades de atenção e gestão, auxiliando os gestores na tomada de decisões em relação à saúde, contribuindo, assim, para a diminuição das assimetrias entre os estados e municípios.

Dessa forma, ao planejar a cooperação com critérios eqüitativos, o Acordo reconhece as diferentes limitações dos seus componentes, traçando ações que possam promover uma maior igualdade de desenvolvimento entre os estados da Região Amazônica e entre as diferentes regiões do País.

(Artigo recebido em junho de 2008. Versão final em setembro de 2008). 


\section{Notas}

${ }^{1}$ O Plano Plurianual sintetiza a orientação estratégica do governo pelo período de quatro anos.

${ }^{2}$ Composto por representantes do Ministério da Saúde (MS), Conselho Nacional de Secretários de Saúde (Conass), Conselho Nacional de Secretarias Municipais de Saúde (Conasems), Fundação Oswaldo Cruz (Fiocruz) e da Plenária Nacional dos Conselhos de Saúde.

${ }^{3}$ Esse grupo foi composto por: Fundação Oswaldo Cruz, Fundação de Medicina Tropical do Amazonas, Universidade Federal do Amazonas, Fundação de Dermatologia Tropical e Venereologia Alfredo da Matta, Instituto Nacional de Pesquisa da Amazônia, Instituto de Pesquisas em Patologias Tropicais de Rondônia e Universidade Federal do Pará.

${ }^{4}$ São elas: 1. malária; 2. micobacterioses; 3 . síndromes febris ictero-hemorrágicas agudas; 4. avaliação de programas e de serviços de saúde; 5 . saúde e ambiente em espaços e em grupos sociais em situação de vulnerabilidade; 6 . saúde: nutrição e alimentação; 7 . fitoterápicos e outros bioativos naturais; e 8. formação de recursos humanos.

${ }^{5}$ Ministério da Saúde, Fundação Oswaldo Cruz, Instituto Evandro Chagas, Universidade Federal do Acre, Laboratório Central de Saúde Pública do Amapá, Fundação de Amparo à Pesquisa do Estado do Amazonas, Fundação de Dermatologia Tropical e Venerologia Alfredo da Matta, Fundação de Medicina Tropical do Amazonas, Instituto Nacional de Pesquisas da Amazônia, Universidade Federal do Amazonas, Universidade Estadual do Amazonas, Instituto de Pesquisas em Patologias Tropicais de Rondônia, Universidade Federal de Rondônia, Centro Universitário do Estado do Pará, Instituto Evandro Chagas, Museu Paraense Emílio Goeldi, Universidade Federal do Pará, Projeto SIPAM, Universidade Federal de Roraima, Fundação de Medicina Tropical de Tocantins e Universidade Federal do Tocantins.

${ }^{6}$ 1. Gestão de CT\&IS, com ênfase em malária; 2. Micobacterioses (micobactérias ambientais, tuberculose e hanseníase); 3. Síndromes febris ictero-hemorrágicas agudas (febre amarela, dengue, hepatites virais, leptospirose); 4. Avaliação de programas e de serviços de saúde; 5. Saúde e ambiente em espaços e em grupos sociais em situação de vulnerabilidade; 6. Saúde: nutrição e alimentação; 7. Fitoterápicos e outros bioativos naturais.

${ }^{7}$ A Guiana Francesa, embora partilhe a Floresta Amazônica, não foi incluída na proposta inicial de formação de Rede Cooperativa por fazer parte do território da União Européia. No entanto, posteriormente, busca-se incorporá-la, considerando que sua inclusão na Rede é de suma importância para superar os obstáculos regionais.

${ }^{8}$ Empoderamento "contempla valores como autonomia, democracia, dignidade da pessoa humana, solidariedade, eqüidade e respeito ao meio ambiente". (31:p.26)

\section{Referências Bibliográficas}

Becker BK; Centro de Gestão e Estudos Estratégicos. Proposta de política de ciência e tecnologia para a Amazônia. Parcerias estratégicas. 2004; (19): 289-309.

- Oficina de trabalho ciência, tecnologia e inovação em saúde na Região Amazônica: relatório. Brasília: Ministério da Saúde; outubro de 2003. 
. Ações de ciência, tecnologia e inovação em saúde para a Amazônia Legal. Rev Saúde Pública. 2007; 41(1): 158-62.

. Cassiolato Je, Lastres Hmm. Globalização e inovação localizada. Brasília: IBICT/MCT; 1999.

A economia política do (des)envolvimento regional contemporâneo. In: Becker D, WitTMann ML. Desenvolvimento regional: abordagens interdisciplinares. Santa Cruz do Sul: UNISC; 2003. p.37-66.

Brasil. Ministério da Saúde. Secretaria-Executiva. Departamento de Apoio à Descentralização. Plano de qualificação da atenção à saúde na Amazônia Legal: plano saúde Amazônia. Brasília: Ministério da Saúde; 2006.

Brasil. Ministério da Saúde. 1a Oficina de Planejamento Regional - construindo uma agenda de saúde para a Amazônia Legal: relatório. Brasília: Ministério da Saúde; agosto de 2003.

Brasil. Ministério da Saúde. Projeto Amazônia: construindo a integração das ações de saúde com investimentos estruturais na região. Brasília: Ministério da Saúde; 2003b.

. $1^{a}$ Oficina do acordo multilateral de cooperação técnico-cientifica em saúde das instituições da Amazônia: relatório. Brasília: Ministério da Saúde, dezembro de 2004.

Brasil. Ministério da Saúde. Fundação Oswaldo Cruz. Centro de Pesquisa Leônidas e Maria Deane. Relatório de atividades do acordo multilateral de cooperação técnicocientífica em saúde das instituições da Amazônia. Manaus: Fundação Oswaldo Cruz; 2005. Versão para circulação restrita. [s.n.]

Brasil. Ministério da Saúde. Conselho Nacional de Saúde. $2^{a}$ Conferência Nacional de Ciência, Tecnologia e Inovação em Saúde (2 ${ }^{a}$ CNCTIS), Brasília, 25 a 28 de julho de 2004. Brasília: Ministério da Saúde; 2004.

Brasil. Ministério da Saúde. Fundação Oswaldo Cruz de Brasília. Rede Pan-amazônica de Ciência, Tecnologia e Inovação em Saúde. Brasília: Fiocruz; 2007.

Brasil. Ministério das Relações Exteriores. Primeira reunião de chefes de estado da comunidade sul-americana de nações - CASA. [documento na Internet]. 2005. [acesso em: 22 de novembro de 2007]. Brasília: MRE; 2005. Disponível em: http:// casa.mre.gov.br/documentos/i-reuniao-de-chefes-de-estado/

Brasil. Ministério do Planejamento, Orçamento e Gestão. Plano plurianual 2004-2007 (PPA 2004/2007). [documento na internet] 2003. [acesso em 8 de outubro de 2007]. Disponível em: http://www.sigplan.gov.br/arquivos/portalppa/41_(menspresanexo).pdf BRASIL. Ministério da Integração Nacional. Política nacional de desenvolvimento regional (PNDR). [documento na internet]. [acesso em 03 de outubro de 2007]. Disponível em: http://www.integracao.gov.br/desenvolvimentoregional/pndr/\#

Plano de desenvolvimento sustentável da Amazônia. [documento na internet]. 2006 Mai. [acesso em 03 de outubro de 2007]. Disponível em: http:// www.integracao.gov.br/publicacoes/diversos/amazonia_sustentavel.asp

Castells M. A sociedade em rede. In: Castells M, Majer RV. A era da informação: economia, sociedade e cultura. São Paulo: Paz e Terra; 1999.

Cavalcanti M, Nepomuceno C. O conhecimento em rede: como implantar projetos de inteligência coletiva. Rio de Janeiro: Elsevier; 2007. 
CGEE. Rede de inovação da biodiversidade da Amazônia. Brasilia:CGEE, dezembro de 2006 (mimeo).

Dallabrida Vr, Siedenberg Dr, Fernández Vr, Desenvolvimento territorial: uma revisão teórica na perspectiva da territorialização e do desenvolvimento. In: WiTTMANN Mu, Ramos Mr. Desenvolvimento Regional: capital social, redes e planejamento. Santa Cruz do Sul: UNISC; 2004. p. 101-103.

Diário Oficial da União, seção 3 - № 206, Anexo 5.

GAdelha CA, Costa L. A política nacional de integração e desenvolvimento das fronteiras: o programa de desenvolvimento da faixa de fronteira - PDFF. In: OliveIRA TCM. Território sem limites: estudos sobre fronteiras. Campo Grande: Universidade Federal do Mato Grosso do Sul; 2005. p. 25-46.

Gallo E, Costa L. Sistema integrado de saúde do Mercosul: Sis-Mercosul uma agenda para integração. Brasília: OPAS; 2004.

Gallo E, Dornelles J, Costa J, Studart VF, Willecke S. Globalização, saúde e desenvolvimento. Divulg saúde debate. 2007; 29:01-17.

Guimarães R. Bases para uma política nacional de ciência, tecnologia e inovação em saúde. Ciência Saúde Coletiva. 2004; 9(2): 375-87.

LEVY P. Inteligencia Colectiva: por una antropología del ciberespacio [periódico na Internet]. 2004 [acesso em 04 de novembro de 2007]. Disponível em: http:// inteligenciacolectiva.bvsalud.org

Machado Lo. Região, cidades e redes ilegais. Geografias alternativas na Amazônia Sulamericana. In: GonçALVES MF, BRANDÃo CA, GALVÃo ACF. Regiões e cidades, cidades nas regiões: o desafio urbano-regional. São Paulo: UNESP: ANPUR; 2003. p. 695-707.

Sítio da rede pan-amazônica de CT\&IS [homepage na internet]. Brasília: Fundação Oswaldo Cruz; [atualizada em 12 de outubro de 2007 ; acesso em 15 de outubro de 2007 ]. Disponível em: http://redepanamazonica.no-ip.org

VAL AL. Formação e fixação de recursos humanos: ações essenciais para a Amazônia. Ciência Cult. 2006; 58(3):41-4.

Wittmann, ML, Dotto DmR, Boff VA. Estruturas organizacionais em rede e desenvolvimento regional: contextualização e complexidades. In: Witrmann ML, Ramos Mr. Desenvolvimento regional: capital social, redes e planejamento. Santa Cruz do Sul: UNISC; 2004. p. 11-36.

ZapATA T. O desenvolvimento territorial endógeno: conceitos, dimensões e estratégias. In: Zapata T, Amorim M, Arns PC. Desenvolvimento territorial a distância. Florianópolis: SEAD/UFSC; 2007. p. 24-34. 


\title{
Resumo-Resumen-Abstract
}

\author{
Acordo Amazônico de Ciência, Tecnologia e Inovação em Saúde: uma experiência \\ de integração Regional \\ Edmundo Gallo, Vivian Studart, Laís Costa, Sandra Willecke e Paulo Lins
}

O presente artigo incorpora a experiência de integração regional amazônica sob a égide do Acordo Amazônico de Ciência, Tecnologia e Inovação em Saúde. Reconhecendo a importância estratégica dessa região detentora de inestimáveis patrimônios em sócio e biodiversidades, o Governo Federal declara, em 2003, a Amazônia uma prioridade para o combate às desigualdades e ao desenvolvimento socioeconômico, articulando, desde então, fóruns com ministérios e instituições regionais, embasados no Programa de Desenvolvimento Sustentável da Amazônia. Com o objetivo de implementar ações para produção de saber científico voltado à realidade local e para a integração da pesquisa básica e aplicada, ensino e tecnologia, incentiva-se a cooperação com outras instituições afins regionais, nacionais e internacionais. Uma dessas ações resultou, em 2004, na formalização do Acordo Multilateral de Cooperação Técnico-Científica em Saúde das Instituições da Amazônia, cuja proposta baseia-se na constituição de uma rede de pesquisa, formação de recursos humanos, cooperação técnica com gestores do SUS e cooperação internacional em saúde, objetivando desenvolver atividades de investigação conjunta para conhecer as realidades sóciossanitárias e epidemiológicas da região e implementar respostas do sistema de saúde e de ciência, tecnologia e inovação.

Palavras-chave: Amazônia, redes de cooperação, integração regional, ciência, tecnologia e inovação em saúde.

\section{Acuerdo Amazónico de Ciencia, Tecnología e Innovación en Salud: una experiencia de integración regional Edmundo Gallo, Vivian Studart, Laís Costa, Sandra Willecke y Paulo Lins}

El presente artículo incorpora la experiencia de integración regional amazónica bajo la tutela del Acuerdo Amazónico de Ciencia, Tecnología e Innovación en Salud. Reconociendo la importancia estratégica de esa región que encierra inestimables riquezas sociales y en biodiversidades, el Gobierno Federal declara, en 2003, que la Amazonia es una región prioritaria para el combate a las desigualdades y al desarrollo socioeconómico, articulando, desde entonces, foros con Ministerios e instituciones regionales, con base en el Programa de Desarrollo Sustentable de la Amazonia. Con el objetivo de implementar acciones para producción de conocimientos científicos direccionados a la realidad local y para la integración de la investigación básica y aplicada, enseñanza y tecnología, se incentiva la cooperación con otras instituciones afines regionales, nacionales e internacionales. Una de esas acciones resultó, en 2004, en la firma del Acuerdo Multilateral de Cooperación Técnico-Científica en Salud de las Instituciones de la Amazonia, cuya propuesta toma por base la constitución de una red de investigación, de formación de recursos humanos, de cooperación técnica con gestores del Sistema Universal de Salud de Brasil - SUS y de cooperación internacional en salud, con miras al desarrollo de actividades de investigación conjunta para conocer las realidades socio-sanitarias y epidemiológicas de la región e implementar respuestas del sistema de salud, de la ciencia, tecnología e innovación.

Palabras-clave: Amazonia, redes de cooperación, integración regional, ciencia, tecnología e innovación en salud 


\section{Amazon Agreement of Science, Technology and Innovation in Health: an Experience of Regional Integration \\ Edmundo Gallo, Vivian Studart, Laís Costa, Sandra Willecke and Paulo Lins}

This article incorporates the experience of regional Amazon integration under the Amazon Agreement of Science, Technology and Innovation in Health. Recognizing the strategic importance of the region, with inestimable patrimony in socio and biodiversity, the Federal Government declared in 2003 that the Amazon is a priority to fight inequality and to foster socioeconomic development, articulating, since then, forum with Ministries and regional institutions, based upon the Program of Sustainable Development of the Amazon. Aiming at implementing actions to production of knowledge within the local reality and for the integration of applied and basic research, teaching and technology; cooperation with other regional institutions, national and international, is motivated. One of the actions resulted, in 2004, in formalizing the Multilateral Agreement of Technical-Scientific Cooperation in Health, with institutions from the Amazon. The proposal is based on the constitution of a research net, formation of human resources, technical cooperation with SUS managers, and international cooperation in health. The objective is to develop joint investigatory activities to acknowledge social-sanitary and epidemiological realities of the region, and to implement answers of the system of health, science, technology and innovation.

Key words: Amazon, cooperation nets, regional integration, science, technology and innovation in health

\section{Edmundo Gallo}

Graduado em Medicina, mestre em Saúde Pública, doutorando em Saúde Pública. Atualmente é Pesquisador da Fundação Oswaldo Cruz - Fiocruz e Consultor de Governos e Organismos Internacionais. É autor, entre outros, de: Razão e Planejamento: Reflexões sobre Política, Estratégia e Liberdade e Planejamento Criativo.

Contato: gallo@fiocruz.br

\section{Laís Costa}

Graduada em Administração de Empresas na Pontifícia Universidade Católica do Rio de Janeiro (PUC-RJ), com mestrado em Development Studies na London School of Economics and Political Science (LSE). Atualmente é pesquisadora do Centro de Pesquisa Leônidas e Maria Deane - CPqLMD, da Fundação Oswaldo Cruz, gerente da Rede Pan-amazônica de CT\&IS por esta Fundação e assessora do gabinete do Departamento de Vigilância Epidemiológica do Ministério da Saúde.

Contato: laiscosta@amazonia.fiocruz.br

Paulo Lins

Bacharel em Relações Internacionais pelo Centro Universitário de Brasília (UniCeub) e bolsista no curso Instrumentos Jurídicos das Relações Econômicas Internacionais e Integração Regional pela Universidade de Barcelona (UB), atualmente é trainee no Setor Executivo da Patri Políticas Públicas, Relações Institucionais e Governamentais.

Contato:pauloale@gmail.com

Vivian Studart

Graduada em Psicologia pela Universidade do Estado do Rio de Janeiro, especialista e mestre em Saúde Pública pela Escola Nacional de Saúde Pública Sérgio Arouca (ENSP/Fiocruz), atualmente é técnica do Grupo de Pesquisa em Saúde Mental e Atenção Básica da Fiocruz Brasília.

Contato: vivian@fiocruz.br

Sandra Willecke

Graduada em Relações Internacionais, Mestre em Gestão e Políticas Públicas e Doutoranda em Saúde Pública. Atualmente é Assessora da Vice-Presidência de Produção e Inovação em Saúde da Fundação Oswaldo Cruz.

Contato: willecke@fiocruz.br 